\title{
Cannabinoids in cancer treatment settings
}

\author{
Thomas Strouse, MD
}

$\mathrm{T}$ he legalization of cannabis is one element of an evolving and multidimensional discourse in the United States. That discourse includes disputes about federal law versus state and individual rights; public misunderstandings about the basic tenets of clinical science, such as concepts of causality and how we weigh evidence about treatment effectiveness; and, in an increasingly consumer-driven health care climate, even the role of physicians in recommending treatments for symptoms and diseases. Whether we like it or not, we who work in cancer find ourselves engaged in the practical consequences of these debates. What is a drug? What does it mean for health care professionals to try to discuss the science of the treatment efficacy of substances, such as most "medical marijuana," that have not been approved by the US Food and Drug Administration (FDA)? How do we find ourselves here?

This commentary is intended to help readers crystallize their own thinking in the face of rapidly emerging new evidence. In a previous editorial, ${ }^{1}$ I attempted to provide commentary on evidence-based uses of cannabinoids in palliative care and hospice; here, the discussion is broadened to include active treatment settings as well.

Much of contemporary medical practice remains palliative - that is to say, physicians and other healers often see and treat patients with chronic, burdensome conditions that are not likely to be cured. Many of those patients suffer from symptoms that are not "fixed" by either disease-modifying or standard palliative treatments. It makes sense then, that patients with such conditions might choose - with or without the involvement of a physician responsible for their longitudinal care - to explore a range of alternative and/or integrative options, including medical marijuana, for symptom management or other efforts to improve disease-related quality of life. What is the state of the science regarding cannabinoids for symptom management in medical illness? How should we be counseling our patients? What kind of inferences can be drawn by clinicians in the service of trying to help our patients translate information from the available clinical science to the bedside and the counter at the marijuana dispensary? What possible jeopardy awaits physicians who endorse medical marijuana use by their patients?

\section{Definition of terms}

In this commentary, marijuana, medical marijuana, and cannabis refer to naturally grown plant materials that are not approved or regulated by the FDA and are procured by patients in a variety of forms (edible, drinkable, volatile) from legal marijuana dispensaries or street suppliers. Cannabinoids refers to 3 chemical classes of compounds: naturally occurring molecules found in the cannabis plant; synthesized molecules, whose structure is based on tetrahydrocannabinol (THC); and the endocannabinoids, which are produced in the CNS of most animals. Pharmaceutical cannabinoids refers to those cannabinoids that have demonstrated safety and efficacy in the treatment of specific clinical problems and have been approved by a national regulatory agency such as the FDA for manufacture and sale based on a physician's prescription. In the latter case, companies that legally produce pharmaceutical cannabinoids are subject to the same manufacturing standards for safety, purity, and content required by the FDA (or its counterpart in other countries) for other drugs and devices.

\section{The big picture}

In July 2014, New York became the 23rd state to legalize personal marijuana possession and its consumption for putative medical purposes. A few days later, the New York Times ran a full-page advertisement sponsored by Leafly, a website that provides information about cannabis, to kick off the site's Just Say Know campaign congratulating the state on its progressive action and endorsing the use of cannabis for medical symptom management. Drawing on pseudoscientific labeling, the ad featured Ian, who "chose an Indica cannabis strain to relieve his multiple sclerosis (MS) symptoms," and Molly, who "while fighting cancer ... preferred a Sativa cannabis." ${ }^{2}$ There was no evidence in the ad to support the claims that cannabis works for either of those indications. Perhaps the advertisers were confident that popular beliefs about a product are sufficient.

The legalization of medical marijuana in New York State generated journalistic and political responses as well. The newspaper's editorial board published a weeklong series of daily editorials - some of which called on the US

JCSO 2016;14:1-5. C2016 Frontline Medical Communications. DOI 10.12788/jcso.0212. 
government to repeal its ban on marijuana, which dates back to the 1970 Controlled Substances Act. More recently, Bernie Sanders, a US senator and Democratic presidential candidate, joined the call for repealing the ban, and in late October 2015 he introduced the Ending Federal Prohibition of Marijuana Act, a Senate bill that would allow states to legalize recreational and medical marijuana in the manner of tobacco and alcohol. ${ }^{3}$

Most of the American public seems to be ready for the legalization of doctor-supervised medical marijuana. In a January 2014 ABC News poll, 86\% of respondents favored legalization for "seriously ill" patients. In addition to the aforementioned 23 states that have passed medical marijuana laws intended to decriminalize possession for personal use or "legitimate" medical uses, Colorado and Washington state have also legalized the sale and possession of marijuana for personal recreational use. However, in a recent article by Richter and Levy, the authors warned that the marijuana industry is not likely to be any more concerned with the public's health or any less voracious in its business practices than the tobacco industry was during the 19 th and 20 th centuries. ${ }^{4}$

\section{The current armamentarium}

At the time of this writing, there were 2 FDA-approved cannabinoid drugs available for prescription in the United States: dronabinol, a synthetic THC compound; and nabilone, a semisynthetic analogue of THC that is about 10 times more potent than dronabinol. ${ }^{5}$ Both of the drugs have been approved for chemotherapy-associated nausea and vomiting; dronabinol is also approved for HIVassociated anorexia/wasting, although the evidence for the latter indication is slim and the problem is much rarer since the advent of antiretroviral drugs. Both dronabinol and nabilone have been studied as possible treatments for other symptoms; though each has shown some efficacy as an adjuvant analgesic, the sedating and psychotropic properties of both agents tend to limit their utility.

Nabiximols, an oral spray that is a near-racemic mixture of THC and cannabidiol (CBD), the 2 main active ingredients in the marijuana plant, has been approved in Canada for opioid-resistant, treatment-refractory cancer pain, and MS-associated spasticity and central pain. In the United Kingdom, Spain, and New Zealand, it has been approved for MS-associated spasticity. It is undergoing phase 3 trials in the United States for cancer pain and may soon be available here.

All other medical marijuana products ingested by patients in the United States are unregulated by the FDA and therefore with uncertain chemical content.

\section{Efficacy}

In a recent Cochrane-style meta-analysis, ${ }^{6}$ investigators assessed the quality of the evidence on the benefits and adverse events (AEs) of cannabinoids in the treatment of chemotherapy-induced nausea and vomiting (CINV), appetite stimulation in HIV/AIDS, chronic pain, spasticity from MS or paraplegia, depression, anxiety, sleep problems, psychosis, glaucoma, or Tourette syndrome. In all, 79 randomized, controlled trials were identified, involving 6,462 patients. The authors concluded that there was moderate quality evidence to support the use of cannabinoids for chronic pain and spasticity. They described as "low" the quality of the evidence supporting the efficacy of cannabinoids for CINV, HIV-associated wasting, sleep problems, and Tourette syndrome. This latter finding is particularly striking because it was that "low" quality evidence that was sufficient to achieve FDA approval for dronabinol and nabilone cannabinoid pharmaceuticals. A companion clinical review article to the analysis offered a clear summary of practical clinical and legal considerations related to medical marijuana in the United States. ${ }^{7}$

The American Academy of Neurology also recently published a review on medical marijuana and it concluded that cannabinoids, particularly nabiximols, may yield marginal benefit in patients with MS for spasticity, central pain, and urinary symptoms, ${ }^{8}$ but identified little utility in other neurologic conditions. Friedman and Devinsky ${ }^{9}$ provided a scholarly summary of the evidence for treatment of epilepsy with cannabinoids. Although they acknowledged preclinical and preliminary and/or anecdotal clinical data, they emphasized the importance of doing standard double-blind trials to help improve the state of knowledge. In another recent review, the authors concluded that marijuana has equivocal effects on generic sleep problems, with some small benefit incurred for pain patients with sleep disturbance. $^{10}$

There is some emerging evidence in pain management that cannabinoids may contribute to reversing opioidassociated hyperalgesia, ${ }^{11}$ may work synergistically to allow lowered opioid dosing, ${ }^{12}$ and may have unique efficacy in the prevention and/or treatment of chemotherapy-induced peripheral neuropathic pain. ${ }^{13}$

\section{Safety}

Volkow and colleagues from the National Institute on Drug Abuse in Bethesda, MD, recently published an excellent review on the health risks of recreational marijuana. ${ }^{14}$ Recreational use of cannabinoids is particularly dangerous for the developing brains of young people and for individuals with existing substance abuse problems and other mental illnesses. Regular use of marijuana can hasten or unmask psychotic illnesses, and it has been associated with diminished IQ. Individuals who use cannabinoids chronically can develop addiction and physical dependence, and there is a well-described withdrawal syndrome. Chronic marijuana use is also associated with increased risk for dropping out of school, overall diminished life satisfaction 
and achievements, and chronic bronchitis. Short-term use of (presumably high THC-containing) recreational marijuana impairs memory, motor coordination, and judgment. All of these are highly concerning findings.

A recent high-quality Canadian prospective cohort study assessed the safety of cannabis used for chronic noncancer pain in a year-long trial. ${ }^{15}$ All of the participants had chronic pain; 215 used (or had used) cannabis for pain, and 216 controls did not use it. The investigators reported that active consumers of cannabis did not have a greater risk for serious AEs, compared with the controls (adjusted incidence rate ratio, 1.08), but that they were at greater risk for nonserious AEs (adjusted incidence rate ratio, 1.73). It is interesting to note that at the end of the trial, both active cannabis users and controls showed improved neurocognitive functioning. Although the high drop-out rate $30 \%$ over 12 months) and high baseline frequency of experienced cannabis use (66\%) raise questions about the generalizability of the data, the findings suggest that for patients with chronic noncancer pain, cannabis use of up to $2.5 \mathrm{~g}$ daily does not seem to confer significant added safety risks.

There is evidence about the public health consequences of widespread cannabis legalization. Recent study findings have demonstrated a two-fold increase in the frequency of marijuana-positive drivers in fatal auto crashes in Colorado since the legalization of cannabis in the state, with no increase in alcohol-related incidents in the same period. ${ }^{16}$ Balanced against these individual and public-safety concerns is a recent finding in state-by-state studies correlating significant reductions in opioid overdose deaths after the enactment of medical marijuana laws. ${ }^{17}$ Although the nature of this correlation remains uncertain, it raises interesting public policy questions and emphasizes the need for further study.

I have argued elsewhere that the concerning safety findings outlined above may be less important for clinical decision-making in a patient with advanced or terminal medical illness who agrees not to drive automobiles or operate other potentially dangerous machinery after they have used marijuana. For middle-aged and older adults with foreshortened life expectancy due to medical illness, both the physician and the patient might conclude that the potential behavioral and intellectual toxicities of cannabinoids could tilt the risk-benefit scale differently than they would for a younger patient with chronic but not life-limiting medical illness who expects to try to compete in the workforce, operate heavy machinery in public venues, parent dependent children, and learn new life and job skills.

The point of this comparison is to underscore that, all other things being equal, the thoughtful consideration of the potential benefits of cannabinoids for a given patient require the perspectives of the treater (if there is one), the patient, and the family; and consideration of the specific clinical circumstances, the patient's disease state and expected natural history, personal developmental state, life aspirations, and other factors. Put differently, an optimal approach to this decision includes a careful risk-benefit discussion and continued close clinical oversight.

\section{Reliability and reproducibility of effects}

There is at best inconsistent evidence that the emerging marijuana industry has made efforts to conduct qualityassurance activities. Some dispensaries, for example, promise that they measure and warrant the chemical composition of each batch of their products. However, a reasonable generalization about the current state of affairs is that the cannabis our patients purchase at the local cooperative will likely contain uncertain concentrations of THC, $\mathrm{CBD}$, and other compounds, despite what the "label" says. For example, a recent small study of marijuana edibles (75 products randomly purchased from internet-listed dispensaries in San Francisco, Los Angeles, and Seattle) showed accurate labeling of THC-CBD content in only $17 \%$ of the products. ${ }^{18}$ The majority of the products $(60 \%)$ were "overlabelled" (ie, at least 10\% less cannabinoid content than claimed), whereas $23 \%$ were "underlabelled" (at least 10\% more cannabinoid content than labeled). Los Angeles dispensaries showed a propensity to underlabel $(P=.01)$, compared with dispensaries in the other cities.

These findings underscore an important reality that is often misunderstood by patients who may not be familiar with the rigors of FDA-approved pharmaceutical manufacturing, which is that most discussions about medical marijuana as a drug therapy represent a leap of faith that the patient has actually received what he/she thinks was purchased. In addition to undermining efforts at a discussion about the reliability and reproducibility of effects, this fact raises a host of other considerations, including issues around basic product safety (are adulterants, congeners, contaminants, insecticides present?), dose-related concerns (eg, little or no pharmacologic effect at one end, and drugrelates toxicities at the other), and possible variation from batch to batch in the product's pharmacologic effects. It also adds an additional level of uncertainty to any efforts by the clinician to consider, discuss, and/or counsel patients about "dose," drug-drug interactions, and other routine clinical issues that might arise around the prescription or endorsement of a new treatment. In addition, the route of administration raises additional levels of uncertainty: bioavailability varies significantly depending on whether cannabis is smoked, vaporized, or orally ingested. ${ }^{19}$

\section{Take home}

Some patients with difficult-to-manage symptoms may benefit from using cannabinoids. Public policy and law enforcement practices related to medical uses of cannabinoids should be governed by science. ${ }^{20}$ Since there is a paucity of clinical trial evidence for the superiority of cannabi- 
noids (in any form) over approved drug therapies, it makes sense for physicians to agree to support their patients to trial medical marijuana when standard treatments are not helping enough. Cannabinoids may also be reasonable options when patients for personal reasons prefer them as first-line over other standard treatments, although it is difficult to provide advice to physicians about how to participate in such activities.

In recommending an approach to determine which patients might be appropriate candidates for physicianendorsed cannabinoids, Hill ${ }^{7}$ has identified 5 commonsense criteria:

1. The patient should have a medical condition for which adequate clinical trials have shown that cannabis has efficacy;

2. The patient should have failed first- and second-line noncannabinoid pharmacotherapies;

3. The patient should have failed an FDA-approved cannabinoid (dronabinol or nabilone);

4. The patient should have no known substance abuse or psychotic illness, and no unstable primary mood or anxiety disorder;

5. The patient should live in a state where medical marijuana is legal.

Beyond Hill's commonsense suggestions, I offer the following 10 addenda:

1. There is reasonably good evidence that cannabinoids may help for some forms of chronic (particularly neuropathic) pain and MS-related spasticity. Cannabinoid use is also accepted in the treatment of CINV and cachexia associated with cancer and HIV, although the science in support of these uses is not very strong. The evidence that cannabinoids may help for other symptoms, such as sleep problems, depression, Tourette syndrome, or refractory seizures, is much weaker.

2. There are FDA-approved cannabinoid prescription formulations that should probably be "first-line agents" in physician-supervised cannabinoid trials. All parties will then know precisely the chemical composition of what is being ingested and there will be clear evidence that federally (FDA-approved) legal approaches were trialed, although as I've already noted, the utility of these agents is often limited by their side-effects. Physicians can prescribe these.

3. Nonprescribing actions by physicians for non-FDA approved medical marijuana vary by state but are generally defined in the laws as endorsements, attestations, or certifications of the possible efficacy of medical marijuana for a particular problem or symptom. Not all physicians will be comfortable signing such a certification (see item 9). In California and many other states, there are physicians who are self- identified as being available to perform case reviews and to complete the attestation paperwork that allows patients to purchase a medical marijuana identity card, which is then used to gain admittance to and purchase from a dispensary.

4. For patients who wish to purchase and ingest medical marijuana with oversight by a continuity-of-care physician, the physician should emphasize that there is a measure of uncertainty about whether the chemical composition of what they (patients) believe they are purchasing is in fact what they are being sold. This uncertainty also makes more speculative efforts to link patients' purchased medical marijuana products to published trials data, and therefore to give standard, informed medical advice.

5. Clinicians should warn patients about the known risks associated with cannabinoids, with the goal of extending the conversation into a risk-benefit discussion. Risk review should extend to friends and family, including children, who may purposefully or inadvertently be exposed to the patients' medicine.

6. With these risks in mind, we should instruct patients to safeguard all cannabinoids as they would with opioids, benzodiazepines, psychostimulants, and other controlled substances. In the absence of compelling data to the contrary, we should also be providing routine warnings to our patients as we would if they were starting on any other CNS-active medicine: care about fall risk, operating automobiles and heavy machinery, and co-administration with other CNS-active agents, including alcohol.

7. Available evidence does not support a common cultural notion that cannabinoids as medicine are "good for whatever ails you." Some people believe this to be true, however, and there is little doubt that in the unregulated world of the dispensary, all manner of health claims are being made. We probably need to consider and respond to such "belief systems" as we would any other sociocultural views that seem to exist outside or beyond the available scientific evidence: respectfully, nonjudgmentally, and professionally. At the same time, we should make efforts to help our patients think critically about these kinds of assertions, particularly if they are leading our patients to eschew evidence-based, disease-modifying treatments in ways that seem to be risky.

8. If we are going to endorse or tacitly support medical marijuana use by our patients, we need to develop basic competence in recognizing the toxicities associated with it, such as intoxication, abuse, withdrawal states, and other side effects.

9. Where we encounter problematic use of cannabinoids - use that constitutes abuse or dependence - we should be prepared to identify the problem, explore 
its context and consequences, and advocate for appropriate treatment, just as we would for our patients who might develop similar problems with other prescribed substances or alcohol.

10. Physicians who want to avoid getting stuck between conflicting state and federal laws on marijuana (which is still inexplicitly classified under Federal law as a Schedule I drug, meaning that it has no medical use and high abuse potential) should not participate as partners or shareholders in the rapidly expanding marijuana production and dispensary industry. It seems unlikely in this era that physicians would be harassed by the Drug Enforcement Administration (DEA) for simply talking with their patients about potential beneficial uses of medical marijuana, or completing an attestation form, but in some states physicians who have served as medical officers or dispensary board members have been intimidated by DEA agents. ${ }^{21}$

\section{References}

1. Strouse TB. Pot in palliative pare: what do we need to know? J Pall Med. 2015;18:7-10.

2. New York Times, Sunday, August 3, 2014.

3. Devaney T. Sanders offers bill to legalize marijuana. http://thehill. com/regulation/259161-sanders-pushing-bill-to-legalize-marijuana. Published November 4, 2015. Accessed November 5, 2015.

4. Richter KP, Levy S. Big marijuana - lessons from big tobacco. N Engl J Med. 2014;371:399-401.

5. Lemberger L, Rubin A, Wolen R, et al. Pharmacokinetics, metabolism, and drug- abuse potential of nabilone. Cancer Treat Rev. 1982;9(suppl B):17-23.

6. Whiting PF, Wolff RF, Deshpande S, et al. Cannabinoids for medical use: a systematic review and meta-analysis. JAMA. 2015;313:2456-2473.

7. Hill KP. Medical marijuana for treatment of chronic pain and other medical and psychiatric problems: a clinical review. JAMA. 2015;313:2474-2483.

8. Koppel BS, Brust JC, Fife T, et al. Systematic review: efficacy and safety of medical marijuana in selected neurologic disorders: report of the Guideline Development Subcommittee of the American Academy of Neurology. Neurology. 2014;82:1556-1563.

9. Friedman D, Devinsky O. Cannabinoids in the treatment of epilepsy. N Engl J Med. 2015;373:1048-1058.

10. Gates PJ, Albertella L, Copeland J. The effects of cannabinoid administration on sleep: a systematic review of human studies. Sleep Med Rev. 2014;18:477-487.

11. Cichewicz DL, McCarthy EA. Antinociceptive synergy between delta(9)THC and opioids after oral administration. J Pharmacol Exp Ther. 2003;304:1010-1015.

12. Russo EB, Hohmann AG. Role of cannabinoids in pain management. In: Deer TR, Leong MS, Buvanendran A, Gordin V, Kim PS, Panchal SJ, Ray AL, eds. Comprehensive treatment of chronic pain by medical, interventional, and integrative approaches. 1st ed. New York, NY: Springer-Verlag; 2013: 181-198.
Some physicians who care for patients with chronic illnesses and associated significant symptom burden take a "don't ask, don't tell" position on medical marijuana. Despite its convenience and tidiness, this is an increasingly untenable position. Medical marijuana and cannabinoid pharmaceuticals seem to be here for the duration: there is a credible evidence base for their efficacy, they are widely available, and they are used widely. Conventional, approved treatments are imperfect, and patients and families are often desperate to find alternatives.

Oncologists should develop basic familiarity with the concepts outlined in this editorial, and should be prepared to inquire about cannabinoid use by their patients. Depending on the outcomes of such an inquiry, oncologists should then be ready to engage in more detailed discussion and decision making, or to assist their patients in getting access to competent consultation from others. "Don't ask, don't tell" serves no one well.

13. Lynch ME, Cesar-Rittenberg P, Hohmann AF. A double-blind, placebo-controlled crossover pilot trial with extension using an oral mucosal cannabinoid extract for treatment of chemotherapy-induced neuropathic pain. J Pain Symptom Manage. 2014;47:166-173.

14. Volkow ND, Baler RD, Compton WM, Weiss SRB. Adverse health effects of marijuana use. N Engl J Med. 2014;370:2219-2227.

15. Ware MA, Wang T, Shapiro S, et al. Cannabis for the management of pain: assessment of safety study (COMPASS). J Pain. 2015;16:1233-1242.

16. Salomunson-Sautel S, Min SJ, Sakai JT, Thurstone C, Hopfer C. Trends in fatal motor vehicle crashes before and after marijuana commercialization in Colorado. Drug Alcohol Depend. 2014;140:137-144.

17. Bachhuber MA, Saloner B, Cunningham CO, Barry CL. Medical cannabis laws and opioid analgesic overdose mortality in the United States, 1999-2010. JAMA Intern Med. 2014;174:1668-1673.

18. Vandrey R, Raber JC, Raber ME, Douglass B, Miller C, BoonMiller MO. Cannabiniod dose and label accuracy in medical cannabis products. JAMA. 2015;313:2491-2493.

19. Borgelt LM, Franson KL, Nussbaum AM, Wang GS. The pharmacologic and clinical effects of medical cannabis. Pharmcotherapy. 2013:33;195-209.

20. Carter GT, Flanagan AM, Earleywine M, Abrahms DI, Aggarwal SK, Grinsppon L. Cannabis in palliative medicine: improving care and reducing opioid-related morbidity. Am J Hospice Pall Med. 2011;28:297-303.

21. Annas GJ. Medical marijuana, physicians, and state law. N Engl J Med. 2014;371:983-985. 